\title{
Introduction to environmental engineering by Stefan Franzle, Bernd Markert, and Simone Winschmann, published by Wiley VCH (2012)
}

\author{
Vinod Kumar Gupta
}

Received: 5 November 2012 / Accepted: 29 November 2012 /Published online: 9 January 2013

(C) Springer-Verlag Berlin Heidelberg 2012

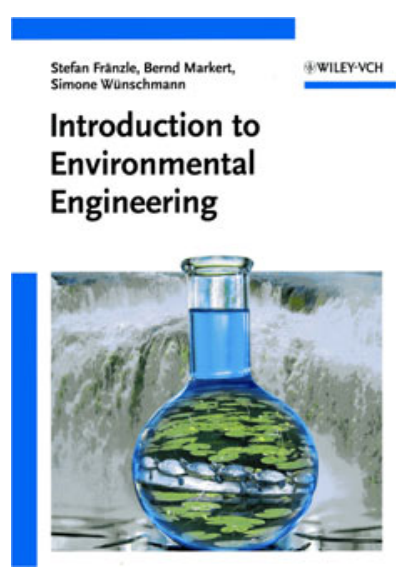

Bibliography

Introduction to Environmental

Engineering

Stefan Fränzle, Bernd Markert,

Simone Wünschmann

Wiley-VCH, Weinheim

ISBN: 978-3-527-32981-6

Softcover, 420 pages

February 2012, €59.

Introduction to Environmental Engineering by Fränzle, Markert, and Wünschmann is another welcome addition to a series of books in the field of environmental science and technology. This is a difficult job and authors have done a fairly good treatment with regard to introduction to the basics of pollution, terms therein used, and methodologies that are adopted to achieve clean environment.

The book consists of four chapters and gives an excellent overview of almost all aspects of environmental science and engineering and is good material to understand this difficult

Responsible editor: Philippe Garrigues

\section{K. Gupta $(\square)$}

Department of Chemistry, Indian Institute of Technology Roorkee, Roorkee 247667 Uttarakhand, India

e-mail: vinodfcy@iitr.ernet.in area for a beginner. The authors start with Chapter 1 where they introduce and define various commonly used terms in chemistry and engineering of environment. In Chapter 2, the authors deal with three important compartments of environmental structure, function, and chemistry. Each environmental compartment is discussed in detail including the material interactions and options to clean them.

Chapter 3 deals with innovative technologies that may be used for cleaning operations. The techniques based on some basics properties - precipitation, adsorption, and immobilization to prevent spread of hazardous materials are discussed. Authors have liberally used pourbaix diaframs, kinetics, and speciation concept to highlight the intricacies of apparently simple technologies.

In Chapter 4, the last chapter, authors have dealt with some aspects of state of art in environmental science and technology with particular reference to bioindicators/biomonitors, EDTA, and diclofenac. The use of plants as bioindicators/biomonitors has been discussed and its efficiency has been pointed out by a comparison with instrumental measurements. The environmental contamination assessment and alleviation of pollution by bioremediation and phytoremediation has been beautifully introduced.

Beside these pollution cleaning technologies discussed, the book gives a detailed introduction to two widely used and stable chemicals EDTA, diclofenac. The properties including adverse effects and toxicity are discussed and technology for their removal is detailed.

As the book has an integrated approach dealt with both the elementary and fairly advanced aspect of environmental science and technology, it can be taken as reference material for researchers and consultants working in the area. For further reading, important and useful references are also included in the end. 